\title{
Cardiac interventions in patients with achondroplasia: a systematic review
}

\author{
Amrita Sukhavasi ${ }^{1}$, Thomas J. O'Malley ${ }^{1}$, Elizabeth J. Maynes ${ }^{1}$, Jae Hwan Choi ${ }^{1}$, Jonathan S. Gordon ${ }^{1}$, \\ Kevin Phan ${ }^{2}$, Vakhtang Tchantchaleishvili ${ }^{1}$ \\ ${ }^{1}$ Division of Cardiothoracic Surgery, Thomas Jefferson University, Philadelphia, Pennsylvania, USA; ${ }^{2}$ Southwest Sydney Clinical School, University \\ of New South Wales (UNSW), Liverpool Hospital, Sydney, Australia \\ Contributions: (I) Conception and design: A Sukhavasi, V Tchantchaleishvili; (II) Administrative support: V Tchantchaleishvili; (III) Provision of \\ study materials or patients: V Tchantchaleishvili; (IV) Collection and assembly of data: A Sukhavasi, EJ Maynes, JH Choi; (V) Data analysis and \\ interpretation: A Sukhavasi, EJ Maynes, JH Choi, JS Gordon; (VI) Manuscript writing: All authors; (VII) Final approval of manuscript: All authors. \\ Correspondence to: Vakhtang Tchantchaleishvili, MD. Assistant Professor of Surgery, Division of Cardiothoracic Surgery, Thomas Jefferson University, \\ 1025 Walnut St, Suite 607, Philadelphia, PA 19107, USA. Email: Vakhtang.Tchantchaleishvili@jefferson.edu.
}

\begin{abstract}
Patients with achondroplasia and other causes of dwarfism suffer from increased rates of cardiovascular disease relative to the remainder of the population. Few studies have examined these patients when undergoing cardiac surgery or percutaneous intervention. This systematic review examines the literature to determine outcomes following cardiac intervention in this unique population. An electronic search was performed in the English literature to identify all reports of achondroplasia, dwarfism, and cardiac intervention. Of the 5,274 articles identified, 14 articles with 14 cases met inclusion criteria. Patientlevel data was extracted and analyzed. Median patient age was 55.5 [interquartile ranges (IQR), 43.8, 59.8] years, median height 102.0 [98.8, 112.5] cm, median BMI 32.1 [27.0, 45.9], and 57.1\% (8/14) were male. Of these 14 patients, nine had the following documented skeletal abnormalities: $66.7 \%(6 / 9)$ had scoliosis, $66.7 \%(6 / 9)$ had kyphosis, 11.1\% (1/9) had lordosis, 11.1\% (1/9) pectus carinatum and 11.1\% (1/9) spinal stenosis. Coronary artery disease was present in 53.8\% (7/13), and 30.8\% (4/13) patients previously suffered a myocardial infarction. Of the eight patients who underwent cardiac surgery, $37.5 \%$ (3/8) underwent multivessel coronary artery bypass grafting, 37.5\% (3/8) underwent aortic valve replacement, $25.0 \%$ (2/8) underwent type A aortic dissection repair, and the remaining 12.5\% (1/8) underwent pulmonary thromboendarterectomy. Six patients underwent percutaneous intervention. Median cardiopulmonary bypass time was 136.5 [110.0, 178.8] minutes. Median arterial cannula size was 20.0 [20.0, 24.0] Fr. Bicaval cannulation was performed in all cases describing cannulation strategy (5/5). Median superior vena cava cannula size was 28.0 [28.0, 28.0] Fr, and inferior vena cava cannula size was 28.0 [28.0, 28.0] Fr. No mortality was reported with a median follow up time of 6.0 [6.0, 10.5] months. In conclusion, Common cardiac procedures can be performed with reasonable safety in this patient population. Operative adjustments may need to be made with respect to equipment to accommodate patient-specific needs.
\end{abstract}

Keywords: Achondroplasia; dwarfism; outcomes; cardiac surgery; cardiac intervention

Submitted Oct 26, 2019. Accepted for publication Jan 18, 2020.

doi: $10.21037 /$ jtd.2020.02.05

View this article at: http://dx.doi.org/10.21037/jtd.2020.02.05

(c) Journal of Thoracic Disease. All rights reserved. 


\section{Introduction}

Achondroplasia and other causes of dwarfism represent a minority of the overall population but only have slightly shorter mean lifespans than non-dwarfs, 61 versus 71 years (1-3). With fairly long life spans, dwarfs are likely to suffer from similar comorbidities as that of aging general population. This population represents 0.36 to 0.60 per 10,000 live births, a minority group, but one that must be considered with respect to interventions that may be required of all aging groups (3). In particular, dwarfs have an increased risk for cardiovascular disease and heartdisease related deaths. Between the ages of 25 to 35, dwarfs have a tenfold increase in risk of mortality from heartrelated disease (4). This has been hypothesized to be due to a propensity for arterial occlusion of narrower vessels due to atherosclerosis in individuals of shorter stature (2). Since this population has an increased risk of cardiovascular disease, there is increased likelihood that dwarfs may require percutaneous or invasive intervention to mitigate atherosclerotic disease. This study examines a series of case reports that have noted cardiac interventions in this patient population and evaluates their safety, efficacy, and outcomes.

While there are multiple causes of dwarfism, achondroplasia is the most prevalent, accounting for more than $90 \%$ of short stature individuals (5). It is inherited in an autosomal-dominant fashion, and is fully penetrant. Despite this inheritance pattern, $90 \%$ of cases are sporadic and due to mutations in G1138A or G1138C in the fibroblast growth factor 3 gene on chromosome 4 p16.3 (5). This results in a skeletal dysplasia with small stature, short limbs with rhizomelic proportions, macrocephaly, a small chest, thoracolumbar kyphosis, and lumbar hyperlordosis (5). These anatomic variations can profoundly affect a patient's life but also must be understood by the clinician as procedures will need to be tailored to the patient in order to be successful.

Given these anatomic variations, Dwarfism has been noted to be challenging for multiple medical providers including the anesthetic clinician (6-8). As this population ages, clinicians will need to be prepared to provide necessary procedures to these individuals. Due to the increased cardiovascular risks and a near normal life expectancy, it is possible that people with dwarfism will need to undergo cardiac intervention.

This systematic review examines 14 cases of patients with achondroplasia undergoing cardiac procedures. As far as the authors know, these are the only cases documented in the English medical literature noting individuals with achondroplasia undergoing cardiac intervention. By pooling this data, we hope to evaluate the safety and efficacy of these procedures, especially with the knowledge that this population is particularly vulnerable to cardiovascular disease.

\section{Methods}

\section{Literature search strategy}

Thorough electronic searches were performed in January 2019 using MEDLINE (Ovid SP), Scopus, CCRT, CINAHL, and PubMed. To achieve the maximum sensitivity of the search strategy, the following combined terms were used: "Dwarfism or Achondroplasia or Receptor, Fibroblast Growth Factor, Type 3, or achondroplast", "Limb deformities, congenital, or osteochondrodysplasias, or cartilage, or skeletal dysplasia", "rhizomelic or chondrodysplasia", "cardiac catheterization", "cardiac catheterization or cardiac surgical procedures or cardiac", "heart atria or heart valves or heart ventricles", "aorta or aortic aneurysm", "coronary vessel anomalies", "dwarf", The reference lists of all eligible studies were reviewed for further identification of potentially relevant studies and assessed using the inclusion and exclusion criteria. A PRISMA flow diagram depicting the overall search strategy is provided in Figure 1.

\section{Selection criteria}

Eligible articles for the present systematic review included case reports/series that focused on patients undergoing cardiac intervention with dwarfism. Patients less than 16 years of age were excluded. Reports not published in the English language, abstracts, conference presentations, editorials, reviews and expert opinions were also excluded. Of the 5,274 articles identified, 14 articles met inclusion criteria consisting of 14 patients. Patient-level data were extracted and analyzed.

\section{Data extraction and critical appraisal}

Data were extracted from article texts, tables, and figures (AS, JC). Discrepancies between the reviewers were resolved by discussion and consensus. When data were not available, attempts were made to contact the corresponding 


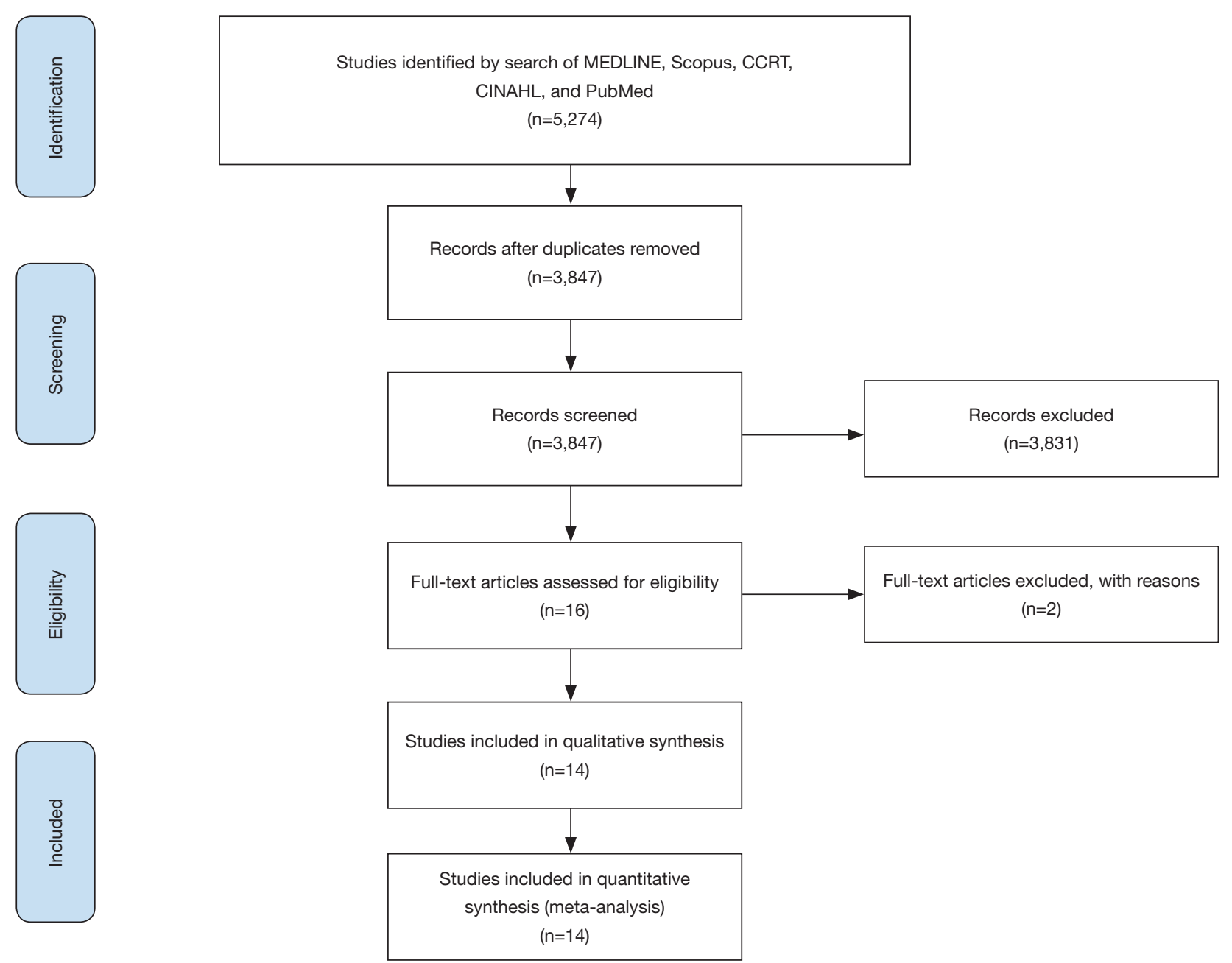

Figure 1 PRISMA schematic diagram of the search strategy. PRISMA, Preferred Reporting Items for Systematic Reviews and MetaAnalysis.

authors to obtain the relevant data for the current study.

\section{Statistical analysis}

Baseline characteristics and demographics were reported using descriptive statistics, including medians and interquartile ranges (IQR) for continuous data and percentages for categorical data.

\section{Evaluation of missing data}

Given the nature of this systematic review with data obtained from individual case reports, not all articles shared the same variables. This discrepancy was evaluated in terms of treatment of missing data and according adjustment of the denominator determining percentage values. Missing data in the routine variables such as demographic variables were treated as missing at random, while variables representing major adverse events were assumed to not have occurred if not reported.

\section{Results}

\section{Study characteristics}

Overall, 5,274 articles were identified in the literature search. Following application of the selection criteria, a total of 14 case reports consisting of 14 patients were included in the analysis. A manual search of references was 
Table 1 Patient demographics

\begin{tabular}{|c|c|}
\hline Variable & Total $(n=14)$ \\
\hline \multicolumn{2}{|l|}{ Patient characteristics } \\
\hline Age, median [IQR] & $55.5[43.8,59.8]$ \\
\hline Male, \% (n/N) & $57.1(8 / 14)$ \\
\hline Height (cm), median [IQR] & $102.0[98.8,112.5]$ \\
\hline Weight (kg), median [IQR] & $40.5[29.5,45.6]$ \\
\hline BSA $\left(\mathrm{m}^{2}\right)$, median [IQR] & $1.1[0.9,1.2]$ \\
\hline BMI $\left(\mathrm{kg} / \mathrm{m}^{2}\right)$, median [IQR] & $32.1[27.0,45.9]$ \\
\hline $\begin{array}{l}\text { No family history of achondroplasia, \% } \\
(\mathrm{n} / \mathrm{N})\end{array}$ & $35.7(5 / 14)$ \\
\hline \multicolumn{2}{|l|}{ Skeletal abnormalities } \\
\hline Scoliosis, \% (n/N) & $66.7(6 / 9)$ \\
\hline Kyphosis, \% (n/N) & $66.7(6 / 9)$ \\
\hline Lordosis, \% (n/N) & $11.1(1 / 9)$ \\
\hline Pectus carinatum, \% (n/N) & $11.1(1 / 9)$ \\
\hline Spinal stenosis, \% (n/N) & $11.1(1 / 9)$ \\
\hline \multicolumn{2}{|l|}{ Medical history } \\
\hline Hypertension, \% (n/N) & $55.6(5 / 9)$ \\
\hline Hypercholesterolemia, \% (n/N) & $55.6(5 / 9)$ \\
\hline Type 2 diabetes, \% (n/N) & $22.2(2 / 9)$ \\
\hline Coronary artery disease, $\%(\mathrm{n} / \mathrm{N})$ & $53.8(7 / 13)$ \\
\hline History of MI, \% (n/N) & $30.8(4 / 13)$ \\
\hline Smoking history, \% (n/N) & $50.0(4 / 8)$ \\
\hline $\begin{array}{l}\text { History of transient ischemic attack, \% } \\
(\mathrm{n} / \mathrm{N})\end{array}$ & $7.1(1 / 14)$ \\
\hline Obstructive sleep apnea, \% (n/N) & $7.1(1 / 14)$ \\
\hline
\end{tabular}

BSA, body surface area; BMI, body mass index; MI, myocardial infarction; IQR, interquartile range.

performed and did not reveal any additional studies.

\section{Baseline characteristics}

Median patient age was 55.5 [IQR, 43.8, 59.8] years, median height was $102.0[98.8,112.5] \mathrm{cm}$, median BMI was 32.1 [27.0, 45.9], and $57.1 \%(8 / 14)$ were male. Of these 14 patients, nine had the following documented skeletal abnormalities: $66.7 \%(6 / 9)$ had scoliosis, 66.7\% (6/9) had kyphosis, 11.1\% (1/9) had lordosis, 11.1\% (1/9) pectus carinatum or $11.1 \%(1 / 9)$ spinal stenosis. With respect to comorbidities placing patients at risk for coronary events, $53.8 \%(7 / 13)$ had coronary artery disease, $55.6 \%(5 / 9)$ had hypertension, 55.6\% (5/9) hypercholesterolemia, and $50.0 \%(4 / 8)$ had a smoking history. $30.8 \%$ (4/13) patients previously suffered a myocardial infarction. Details on baseline characteristics are outlined in Table 1.

\section{Pre-operative characteristics}

Pre-operatively, the median left ventricular ejection fraction was $50 \%[40,60]$. Coronary artery disease was present in $53.8 \%(7 / 13)$ of patients. Right coronary artery stenosis was present in $75.0 \%(6 / 8)$ of patients. This was followed by $71.4 \%(5 / 7)$ of patients with left anterior descending stenosis, $57.1 \%(4 / 7)$ of patients with circumflex stenosis. In total $50.0 \%(3 / 6)$ patients presented with ST segment elevation. With respect to those with valvular disease, $21.4 \%(3 / 14)$ had aortic stenosis. Of these patients, the median maximum aortic valve transvalvular gradient was 114.5 [99.8, 129.2] mmHg. Additional preoperative characteristics are demonstrated in Table 2.

\section{Operative variables}

We examined the patient population who underwent two different sets of interventions: surgery and percutaneous intervention. Of these, $57.1 \%(8 / 14)$ patients underwent surgery and $42.9 \%(6 / 14)$ underwent percutaneous intervention. Within the surgery group, $37.5 \%(3 / 8)$ underwent multivessel coronary artery bypass grafting, $37.5 \%(3 / 8)$ underwent aortic valve replacement, one of which was during a concomitant Type A aortic dissection repair, 25.0\% (2/8) underwent type A aortic dissection repair, and the remaining $12.5 \%(1 / 8)$ underwent pulmonary thromboendarterectomy. Of the percutaneous group, $66.7 \%$ (4/6) underwent percutaneous coronary intervention, $16.7 \%(1 / 6)$ underwent balloon aortic valvuloplasty and balloon pulmonary valvuloplasty, and $16.7 \%(1 / 6)$ underwent micra transcatheter pacing system implantation.

Of the patients who had surgery performed, $100.0 \%$ $(8 / 8)$ underwent cardiopulmonary bypass (CPB) with a median aortic cross clamp time of $91.0[67.5,96.5]$ minutes and a total cardiopulmonary bypass time of 136.5 [110.0, 178.8] minutes. Arterial cannula size was 20.0 [20.0, 24.0] Fr. Bicaval cannulation was performed in all cases $(5 / 5)$. 
Table 2 Preoperative characteristics

\begin{tabular}{lc}
\hline Variable & Total $(\mathrm{n}=14)$ \\
\hline Ejection fraction \% (n/N), median [IQR] & $50[40,60]$ \\
Coronary artery disease & $53.8(7 / 13)$ \\
Right coronary artery stenosis, \% (n/N) & $75.0(6 / 8)$ \\
Left anterior descending stenosis, \% (n/N) & $71.4(5 / 7)$ \\
Circumflex stenosis, \% (n/N) & $57.1(4 / 7)$ \\
ST elevation, \% (n/N) & $50.0(3 / 6)$ \\
Aortic stenosis, \% (n/N) & $21.4(3 / 14)$ \\
Hypertrophic interventricular septum & $66.7(2 / 3)$ \\
Interventricular septum size (mm), median [IQR] & $14.0[13.5,14.5]$ \\
Left ventricular hypertrophy, \% (n/N) & $33.3(1 / 3)$ \\
Maximum aortic valve transvalvular gradient & $114.5[99.8$, \\
(mmHg), median [IQR] & $129.2]$ \\
Aortic valve area (cm $\left.{ }^{2}\right)$, median [IQR] & $0.5[0.5,0.5]$ \\
Pulmonary stenosis, \% (n/N) & $7.1(1 / 14)$ \\
Mean pulmonary artery pressure (mmHg), & $45.0[39.0,51.5]$ \\
median [IQR] & \\
Preoperative atrial fibrillation, \% (n/N) & $7.1(1 / 14)$ \\
\hline
\end{tabular}

$I Q R$, interquartile range.

Superior vena cava cannula size was 28.0 [28.0, 28.0] Fr with a maximum size of $30 \mathrm{Fr}$, and inferior vena cava cannula size was 28.0 [28.0, 28.0] Fr with a maximum size of $30 \mathrm{Fr}$. With respect to aortic valve replacement, the sizes of the implanted valve prostheses were $16 \mathrm{~mm}$ [14], $20 \mathrm{~mm}$ [15], and $23 \mathrm{~mm}$ [9], respectively. The median aortic root size was $11.0[10.5,31.5] \mathrm{mm}$ with $33.3 \%(1 / 3)$ of patients undergoing aortic root enlargement. In this patient, root enlargement was accomplished by extending the incision through the noncoronary Sinus of Valsalva along the subaortic space and patching it with autologous pericardium (9). Further operative details can be found in Table 3.

\section{Outcomes and complications}

Post-operatively, patients spent a median of $2[1,5]$ days in the intensive care unit with a median hospital length of stay of 7 [5, 15] days. The postoperative aortic valve gradient was $11.0[9.5,13.5] \mathrm{mmHg}$. One patient was centrally cannulated for veno-arterial extracorporeal life support following pulmonary artery endarterectomy. All patients
(14/14) were asymptomatic relative to coronary symptoms at the time of discharge with a median follow up time of $6.0[6.0,10.5]$ months and continued to be asymptomatic at follow up (10/10). None of the cases reported mortality with a median follow up time of $6.0[6.0,10.5]$ months. Additional information regarding postoperative outcomes and complications can be found in Tables 4 and 5 .

\section{Discussion}

Achondroplastic dwarfism represents a clinical challenge for surgeons and clinicians given differences in anatomy and physiology. Given a relatively similar lifespan as the general population and a propensity for early cardiovascular disease, it is not uncommon for these patients to develop long-term health problems and require cardiac intervention. The risk of heart disease is twice as high in achondroplastic dwarfs compared to the general population and three times as high when comparing male achondroplastic dwarfs with the general population (4). With studies noting increased cardiovascular risk in patients with achondroplasia, it stands to reason that these patients will require intervention in the future. These patients require special procedural considerations with respect to anesthesia, operative technique, and postoperative care. These considerations have been evaluated previously with respect to other operations, but cardiac interventions have not been previously examined on a scale larger than case reports. Our data demonstrated excellent survival, minimal complications, and excellent secondary outcomes. It should be noted the inherent bias in obtaining this data from individual case reports which is noted in our limitations discussion.

With respect to achondroplastic dwarfs, considerations had to be made to adjust procedures to differences in patient anatomy and physiology. From an anesthesia perspective, the kyphotic cervical spine, maxillary hypoplasia and megalencephaly can present difficult airway challenges in patients with dwarfism (6). In the case reports that detailed the intubation process, all three cases used a method of video-assisted intubation. Anesthetic literature has also suggested using smaller endotracheal tubes basing the size on the weight of the patient (6). In our patient cohort, $35.7 \%(5 / 14)$ patients required pediatric endotracheal tubes. The remaining nine case reports did not specify the type or size of the endotracheal tube. Following intubation, intravascular access and patient positioning are both challenging due to short limbs, reduced joint mobility, and 
Table 3 Intraoperative characteristics

\begin{tabular}{|c|c|}
\hline Variable & Total $(n=14)$ \\
\hline Surgery, \% (n/N) & $57.1(8 / 14)$ \\
\hline Multivessel CABG, \% (n/N) & $37.5(3 / 8)$ \\
\hline Aortic valve replacement, \% (n/N) & $37.5(3 / 8)$ \\
\hline Type A aortic dissection repair, \% (n/N) & $25.0(2 / 8)$ \\
\hline $\begin{array}{l}\text { Pulmonary thromboendarterectomy, \% } \\
(\mathrm{n} / \mathrm{N})\end{array}$ & $12.5(1 / 8)$ \\
\hline Percutaneous intervention, \% (n/N) & $42.9(6 / 14)$ \\
\hline $\begin{array}{l}\text { Percutaneous coronary intervention, \% } \\
(\mathrm{n} / \mathrm{N})\end{array}$ & $66.7(4 / 6)$ \\
\hline $\begin{array}{l}\text { Balloon aortic valvuloplasty and balloon } \\
\text { pulmonary valvuloplasty, } \%(n / N)\end{array}$ & $16.7(1 / 6)$ \\
\hline $\begin{array}{l}\text { Micra transcatheter pacing system } \\
\text { implantation, } \%(n / N)\end{array}$ & $16.7(1 / 6)$ \\
\hline \multicolumn{2}{|l|}{ Intubation method } \\
\hline Fiberoptic scope, \% (n/N) & $33.3(1 / 3)$ \\
\hline Glidescope, \% (n/N) & $33.3(1 / 3)$ \\
\hline Video laryngoscopy, \% (n/N) & $33.3(1 / 3)$ \\
\hline Pediatric endotracheal tube, \% (n/N) & $35.7(5 / 14)$ \\
\hline Cardiopulmonary bypass, \% (n/N) & $100.0(8 / 8)$ \\
\hline \multicolumn{2}{|l|}{ Temperature } \\
\hline Normothermia $\left(>34^{\circ} \mathrm{C}\right), \%(\mathrm{n} / \mathrm{N})$ & $25.0(2 / 8)$ \\
\hline Mild hypothermia $\left(28.1-34^{\circ} \mathrm{C}\right), \%(\mathrm{n} / \mathrm{N})$ & $12.5(1 / 8)$ \\
\hline $\begin{array}{l}\text { Moderate hypothermia }\left(20.1-28^{\circ} \mathrm{C}\right), \% \\
(\mathrm{n} / \mathrm{N})\end{array}$ & $12.5(1 / 8)$ \\
\hline Deep hypothermia $\left(14.1-20^{\circ} \mathrm{C}\right), \%(\mathrm{n} / \mathrm{N})$ & $25.0(2 / 8)$ \\
\hline Circ arrest time (minutes), median [IQR] & $40.0[29.5,50.5]$ \\
\hline CPB time (minutes), median [IQR] & $136.5[110.0,178.8]$ \\
\hline \multicolumn{2}{|l|}{ Arterial cannulation site } \\
\hline Aorta, \% (n/N) & $60.0(3 / 5)$ \\
\hline Axillary, \% (n/N) & $20.0(1 / 5)$ \\
\hline Femoral, \% (n/N) & $20.0(1 / 5)$ \\
\hline Arterial cannula size (Fr), median [IQR] & $20.0[20.0,24.0]$ \\
\hline Bicaval cannulation & $100.0(5 / 5)$ \\
\hline $\begin{array}{l}\text { Superior vena cava cannula size }(\mathrm{Fr}) \text {, } \\
\text { median }[\mathrm{IQR}]\end{array}$ & $28.0[28.0,28.0]$ \\
\hline $\begin{array}{l}\text { Inferior vena cava cannula size }(\mathrm{Fr}) \text {, } \\
\text { median [IQR] }\end{array}$ & $28.0[28.0,30.0]$ \\
\hline
\end{tabular}

Table 3 (continued)
Table 3 (continued)

\begin{tabular}{lc}
\hline Variable & Total $(\mathrm{n}=14)$ \\
\hline Coronary artery bypass grafting & \\
Vein harvested & \\
Left saphenous, \% (n/N) & $33.3(1 / 3)$ \\
Right and left saphenous, \% (n/N) & $33.3(1 / 3)$ \\
Right saphenous, \% (n/N) & $33.3(1 / 3)$ \\
Vein quality & \\
Normal, \% (n/N) & $66.7(2 / 3)$ \\
Moderate, \% (n/N) & $33.3(1 / 3)$ \\
Artery harvested & $66.7(2 / 3)$ \\
LIMA, \% (n/N) & $33.3(1 / 3)$ \\
LIMA and RIMA, \% (n/N) & \\
Artery quality & $33.3(1 / 3)$ \\
Normal, \% (n/N) & $100.0(2 / 2)$ \\
Aortic valve replacement & \\
Aortic cross clamp time (minutes), \\
median [IQR]
\end{tabular}

CABG, coronary artery bypass grafting; circ, circulation; CPB, cardio-pulmonary bypass; LIMA, left internal mammary artery; RIMA, right internal mammary artery; IQR, interquartile range.

anatomy of the chest (8). Several reports note the use of ultrasound guidance for intravascular access and arterial and vein cut-downs for safe vascular exposure $(10,11)$. These differences from the general population must be considered in preparing for procedures on patients with achondroplasia.

With respect to cardiac surgery interventions in these patients, several operative techniques should be adjusted or considered prior to and during the case. For procedures requiring $\mathrm{CPB}$, smaller cannula sizes were used in general. It should be noted that all five cases where cannulation was documented reported bicaval cannulation. This was likely performed due to crowding from relatively large organs in a 
Table 4 Postoperative characteristics

\begin{tabular}{lc}
\hline Variable & Total $(\mathrm{n}=14)$ \\
\hline Time in ICU (days), median [IQR] & $2[1,5]$ \\
Number of postoperative days in hospital, median & $7[5,15]$ \\
[IQR] & \\
Postoperative aortic valve gradient (mmHg), & $11.0[8.5,13.5]$ \\
median [IQR] & \\
Postoperative complications & $7.1(1 / 14)$ \\
VA ECMO, \% (n/N) & $7.1(1 / 14)^{*}$ \\
Mediastinal bleeding, \% (n/N) & $7.1(1 / 14)^{*}$ \\
Pleural effusion, \% (n/N) & $7.1(1 / 14)^{*}$ \\
Pneumothorax, \% (n/N) & $7.1(1 / 14)^{*}$ \\
Pulmonary embolism, \% (n/N) & $7.1(1 / 14)$ \\
Atrial fibrillation, \% (n/N) & \\
\hline * same patient who had undergone coronary artery bypass \\
grafting. ICU, intensive care unit; IQR, interquartile range; VA \\
ECMO, veno-arterial extracorporeal membrane oxygenation.
\end{tabular}

Table 5 Outcomes

\begin{tabular}{lc}
\hline Variable & Total $(\mathrm{n}=14)$ \\
\hline Asymptomatic at discharge, \% $(\mathrm{n} / \mathrm{N})$ & $100.0(14 / 14)$ \\
Follow up time (months), median $[\mathrm{IQR}]$ & $6.0[6.0,10.5]$ \\
Asymptomatic at last follow up, \% (n/N) & $100.0(10 / 10)$ \\
30-day mortality, \% (n/N) & $0.0(0 / 14)$ \\
Overall mortality, \% $(\mathrm{n} / \mathrm{N})$ & $0.0(0 / 14)$ \\
\hline IQR, interquartile range. &
\end{tabular}

smaller chest cavity. One report noted that surgeons should assess patients for the largest possible cannulation sizes for $\mathrm{CPB}$ to eliminate the possibility of reduced flow that may compromise end-organ perfusion (11). For procedures requiring artery or vein conduit, it should be noted that saphenous vein graft may be challenging to obtain, too short for purposes of a bypass, or unsuitable for bypass. One such report noted smaller length of saphenous vein harvest with moderate quality due to patient anatomy and performed a three-vessel coronary-artery bypass with both right and left internal mammary arteries (10). Another report noted a $29 \mathrm{~cm}$ saphenectomy incision that extended to the patient's ankle (12), and a third noted pre-operative phlebography to assess for vein quality (13). A third report noted unsuitable grafts given the severe musculoskeletal changes (14). Once median sternotomy was performed, reports noted concerns regarding organ crowding with respect to the patient's chest anatomy, but found chest and organ size consistent with the general population $(10,11)$. However, due to some thinning of the sternum, one such report noted closure with absorbable suture in lieu of stainless steel wire (9). With respect to aortic valve replacement, our investigation noted the placement of valves required surgical aortic root enlargement in one case (9) with aortic root dilation in another case (15). These technical considerations must be considered prior to performing any cardiac intervention on patients with achondroplasia.

Aortic procedures in these patients also have unique considerations. Two patients presented with type A dissection and underwent subsequent hemiarch repairs. Due to the small caliber of their major vessels, the feasibility of cerebral perfusion was initially in question. However, the authors did not encounter any difficulty with antegrade selective cerebral perfusion in these patients. One patient underwent central arterial and venous cannulation, while another patient underwent peripheral arterial cannulation but central venous cannulation.

With respect to percutaneous interventions in patients with achondroplasia, our systematic review of available interventions in this population demonstrates safe and effective use of these techniques to alleviate and treat cardiovascular conditions in these patients. Studies have noted technical difficulties with endovascular access of the coronary arteries using the transradial approach due to short limbs, elbow angles and kyphoscoliosis $(16,17)$. Obtaining arterial access has also been noted to be challenging with respect to accessing the femoral vessels, and one study noted ultrasonographic assistance was required (14). Determination of radial versus femoral access should be determined on a case-by-case basis with the two reports of radial access noting their choice was based on complexity of intervention required $(17,18)$. Further studies are necessary to elucidate the benefits and disadvantages of each technique. Although obtaining coronary access was difficult, no challenges with respect to coronary morphology were encountered. In total these six reports noted success at achieving revascularization or elimination of stenosis with minimal complication.

Evaluation of both surgical and percutaneous techniques revealed several suggestions by each of these case reports. Unfortunately, due to the number of cases in total, these recommendations are areas that require further study. For surgical intervention, these case reports suggest the 
following: videoscopic intubation with smaller endotracheal tubes, obtain intravascular access using ultrasonography and arterial cutdown where necessary, use the largest available cannula size that vessels can accommodate with pediatric sized cannulas available, perform preoperative phlebography of saphenous veins if being used as conduits, and if aortic valve replacement is required, consider aortic root enlargement or dilation for appropriate fitting of the replacement valve. With respect to percutaneous intervention, clinicians should be aware of the challenges of coronary vessel cannulation and the relative advantages and disadvantages of radial versus femoral access with respect to patient anatomy using ultrasonography when able for vessel access.

This systematic review has demonstrated performing surgical and percutaneous interventions in dwarfs is both safe and effective though further study is needed to ensure this is not due to a selective publishing of favorable cases. Operative and procedural considerations must be made to ensure success though these can be accomplished with expert care and preoperative planning. Further, a prospective registry would be beneficial to determine best practices. Given the pre-operative concerns of organ size and crowding shared by the articles included in this systematic review, it is possible that this sentiment is shared by other cardiac surgeons. This predicted challenge represents a barrier that may make surgeons hesitant to operate on achondroplastic dwarfs and decline surgical intervention for those who may benefit from an intervention. A prospective registry would further support the findings of this systematic review and help to eliminate operative concerns surgeons may have with respect to this population's anatomy. This systematic review of the available literature suggests these procedures can be accomplished safely and effectively.

\section{Limitations and future directions}

Achondroplasia represents a relatively rare condition and therefore this systematic review encapsulates the minority of this small subgroup of patients undergoing cardiac interventions. Given this, most of the data in this systematic review comes from individual case reports, which are subject to a publication bias. Successful cases are more likely to be reported which is especially true here as these reports show excellent survival and low complication rates. Ideally, a prospective registry would follow patients with the necessary pre-operative characteristics following their intervention. However, a prospective database here is likely to suffer from a lower power given our current literature search only produced 14 of this particular type of patient. Our results with respect to survival and complications should be viewed with the understanding that the cases where patients did not survive may not be reported and therefore our survival is likely overestimated and our complications underestimated.

\section{Conclusions}

Common cardiac procedures can be performed with reasonable safety in patients with achondroplasia. Operative and procedural adjustments may need to be made with respect to equipment and technique to accommodate patient-specific needs.

\section{Acknowledgments}

Funding: None.

\section{Footnote}

Conflicts of Interest: The authors have no conflicts of interest to declare.

Ethical Statement: The authors are accountable for all aspects of the work in ensuring that questions related to the accuracy or integrity of any part of the work are appropriately investigated and resolved.

Open Access Statement: This is an Open Access article distributed in accordance with the Creative Commons Attribution-NonCommercial-NoDerivs 4.0 International License (CC BY-NC-ND 4.0), which permits the noncommercial replication and distribution of the article with the strict proviso that no changes or edits are made and the original work is properly cited (including links to both the formal publication through the relevant DOI and the license). See: https://creativecommons.org/licenses/by-nc-nd/4.0/.

\section{References}

1. Hecht JT, Francomano CA, Horton WA, et al. Mortality in achondroplasia. Am J Hum Genet 1987;41:454-64.

2. Wamala SP, Mittleman MA, Horsten M, et al. Short stature and prognosis of coronary heart disease in women. J Intern Med 1999;245:557-63. 
3. Waller DK, Correa A, Vo TM, et al. The populationbased prevalence of achondroplasia and thanatophoric dysplasia in selected regions of the US. Am J Med Genet A 2008;146A:2385-9.

4. Wynn J, King TM, Gambello MJ, et al. Mortality in achondroplasia study: a 42-year follow-up. Am J Med Genet A 2007;143A:2502-11.

5. Vajo Z, Francomano CA, Wilkin DJ. The molecular and genetic basis of fibroblast growth factor receptor 3 disorders: the achondroplasia family of skeletal dysplasias, Muenke craniosynostosis, and Crouzon syndrome with acanthosis nigricans. Endocr Rev 2000;21:23-39.

6. Mayhew JF, Katz J, Miner M, et al. Anaesthesia for the achondroplastic dwarf. Can Anaesth Soc J 1986;33:216-21.

7. Jain A, Jain K, Makkar J, et al. Case study: Anaesthetic management of an achondroplastic dwarf undergoing radical nephrectomy. South African J Anaesth Analg 2010;16:77-9.

8. Berkowitz ID, Raja SN, Bender KS, et al. Dwarfs: pathophysiology and anesthetic implications. Anesthesiology 1990;73:739-59.

9. Scafuri A, Moscarelli M, Guerrieri Wolf L, et al. Aortic root enlargement for aortic valve replacement in an achondroplastic dwarf. Tex Heart Inst J 2005;32:442-4.

10. Al-Jughiman $M$, Yanagawa B, Rondi K, et al. Acute Type A Dissection Repair in an Achondroplastic Dwarf: Anesthetic, Perfusion, and Surgical Concerns. AORTA

Cite this article as: Sukhavasi A, O'Malley TJ, Maynes EJ, Choi JH, Gordon JS, Phan K, Tchantchaleishvili V. Cardiac interventions in patients with achondroplasia: a systematic review. J Thorac Dis 2020;12(3):998-1006. doi: 10.21037/ jtd.2020.02.05
2014;2:143-6

11. Moriyama S, Hara M, Kaneko Y. Acute type A aortic dissection repair in an octogenarian with achondroplasia: a case report. Surg Case Rep 2018;4:53.

12. Balaguer JM, Perry D, Crowley J, et al. Coronary artery bypass grafting in an achondroplastic dwarf. Tex Heart Inst J 1995;22:258-60.

13. Tagarakis GI, Karangelis D, Baddour AJ, et al. Coronary artery surgery in a man with achondroplasia: a case report. J Med Case Rep 2010;4:348.

14. Kumar V, Kumar V. A case of multivessel PTCA in achondroplasia patient. Egypt Heart J 2017;69:85-8.

15. Baikoussis NG, Argiriou M, Argiriou O, et al. Perceval $S$ aortic valve implantation in an achondroplastic Dwarf. Ann Card Anaesth 2016;19:166.

16. Srinivas SK, Ramalingam R, Manjunath $\mathrm{CN}$. A rare case of percutaneous coronary intervention in achondroplasia. J Invasive Cardiol 2013;25:E136-8.

17. Verma B, Singh A, K Saxena A, et al. Transradial Coronary Angioplasty in an Achondroplastic Patient with Chronic Total Occlusion (CTO): First Case Report. J Cardiovasc Dis Res 2018;9:151-3.

18. Rahman N, Nabi A, Gul I. Sheathless transradial coronary angioplasty in an achondroplasic patient with ST elevation myocardial infarction. BMJ Case Rep 2015;bcr2015212697. 